\title{
New Mathematical model of Retailer-to-Individual Customer Optimal Product Supply Strategies Under False Demand Pattern: Customer Discount Mode
}

\author{
Zhiyi Zhuo \\ Correspondence: Chinese Graduate School, Panyapiwat Institute of Management, Nonthaburi 11120, Thailand. \\ E-mail: zhuozhiyi@pku.org.cn
}

Received: November 4, 2019 Accepted: December 4, 2019 Online Published: January 12, 2020

doi:10.5539/jmr.v12n1p36

URL: https://doi.org/10.5539/jmr.v12n1p36

\begin{abstract}
This paper develops a new mathematical model by using the false demand function and the retailer profit function, to study the retailer-to-individual customer optimal product supply strategies under the false demand pattern, and solve the product profit optimal problem. The research contribution of this paper is to construct the mathematical model of customer discount mode for the retailer-to-individual customer product supply strategies under the false demand pattern. The study results provide a reference for retailers to develop different supply strategies for different types of customers in different demand patterns, enabling them to improve operational performance effectively.
\end{abstract}

Keywords: false demand pattern, product supply strategies, mathematical model

\section{Introduction}

Demand theory since the publication of Marx's "German Ideology" at the end of the nineteenth century (Marx \& Engels, 1965), demand-management research has been continuously heated for for one and a half centuries. Scholars have used quantitative and qualitative tools to study demand management, covering almost all fields. People have explored the theoretical and applied research of demand in various fields, and studied multiple details in these theories and applications, and have formed demand academic research in numerous areas, such as supply chain field, economics field, psychology field, etc. (Maslow, 1943; Morgenstern, 1948; McClelland et al, 1953; Marcuse, 1991; Rachlin et al., 1976; F. Bustinza, C. Parry \& Vendrell-Herrero, 2013). This article studies how companies design and plan product supply strategies based on customer demand, so it is a demand theory study of the consumer product supply chain.

In the field of consumer product supply chains, demand is an essential cognition of end consumers and a vital means to grasp the latest market trends. For example, suppliers cannot accurately grasp the latest market demand information, so they cannot effectively formulate product specifications and sales decisions that meet the latest market trends or fashion trends (Gause \& Weinberg, 2011); retailers can observe product sales to predict Market demand information to make optimal purchasing decisions based on trade-offs between forecast reliability, procurement costs, and the availability of existing resources (Zhang, Duan \& Ma, 2018). Thus, customer demand has gradually become a significant factor affecting product design, manufacturing, and sales.

In the subject literature on product supply chains from demand orientation, Nazari \& Foroud (2013) study the retailer's short and medium-term decision planning under demand uncertainty. Yuan, Gómez \& Rao (2013) studied trade promotion decisions in a manufacturer's retailer channel where retailers face consumer demand uncertainty. Cohen, Lobel \& Perakis (2016) studied the production and pricing decisions of suppliers under uncertain demand based on government subsidies. Özer \& Uncu (2015) studied the time-to-market decision and subsequent sales channel, pricing, and production decisions of companies under uncertain demand. Kim \& Krishnan (2015) studied the interactive impact of product demand uncertainty and product price on online consumer purchase decisions. Moradi et al. (2018) studied the optimization decision model of agile supply chain networks under uncertain demand conditions. Kisialiou, Gribkovskaia, and Laporte (2019) solve the problem of supply vessel planning in upstream offshore oil logistics under uncertain demand. Abdel-Aal \& Selim (2019) study the selective newsvendor problem (SNVP), where the decision-maker selects the optimal set of markets to serve and the optimal order quantity to procure. Weskamp et al. (2019) studied a stochastic mixed-integer linear programming model for decision-makers under demand uncertainty. The model takes into account delivery time, shortage penalty costs, and inventory retention decisions within the scope of tactical planning. Xie et al. (2019) studied advertising outsourcing and production planning for manufacturers with asymmetric advertising costs and uncertain market demand. 
By summarizing the above-related literature, we find that the existing studies are only the results of the uncertainty of demand, and ignore the three different customer demand patterns in the management practice of the supply chain of consumer goods: real pattern, false pattern, and semi-real pattern. In the field of consumer product supply chain, customer demand is the core of product supply. Thus, there are bound to be two factors that influence customer purchase decisions. One is the products that customers must buy during production or in a certain period, such as daily necessities such as oil and cereals. That is a demand that consumers really need and a demand that is necessary to maintain human existence and development. The nature of this need is real demand. The other is that customers have no desire to purchase because they are guided by external forces, which leads to the actual purchase of products. The nature of this demand that affects consumers' purchase behavior due to external factors such as economic benefits and advertising is a false demand. The essence of false demand is the product of ideology and social culture acting on individuals, and it is a pure spirit or psychological phenomenon (Marcuse, 1991). In a study of consumer buying behavior, Crawford (2004) found that the consumer arrived at the mall with a "really needed need" and faced a series of information about his or her "need," eventually, with the increasing product range, consumers have forced to divide requirements into ever smaller elements. This ever-smaller element is a demand that lies between real demand and false demand, that is, semi-real demand. We give an example to explain our theoretical basis. From the simple real case of drinking coffee, it can be seen that the function of quenching thirst is no longer the main factor leading to consumer buying behavior. We found several different levels of functional dimensions for customer needs. The first level of demand is the function of quenching thirst, which is the essential function of drinking coffee. That meets basic physiological needs is real needs. However, modern urban people drink coffee not only to meet the basic physiological needs of quenching thirst. At this time, the demand for the second level, that is, social functions is manifested in the fact that people often meet in coffee shops. Many times people drink coffee together to promote work exchanges and cooperation. At this time, the level of demand for coffee has risen to the function of work. From the discussion of the three dimensions of the above requirements affecting customers' coffee-drinking behavior, it can be found that the essential functional factors can no longer meet the customer's needs, and ideological factors have become the mainstream factors that increasingly affect consumer purchasing behavior (Marcuse, 1991). With the development of the economy and the advancement of science and technology, enterprises have transmitted the false demand awareness to the mass consumers through the influence and manipulation of various media. Here, rich products play a role in indoctrination and manipulation. They cause a false demand consciousness that is difficult to see. Finally, this false demand not only replaces real demand but also becomes the basic lifestyle of consumers. This article studies the retailer based on the customer discount model to design and plan product supply strategies for individual customers under false demand. This field represents an important theoretical gap in the research of the consumer product supply chain and has great theoretical significance.

\section{Variable Functions}

\subsection{False Demand Function}

We study such a simple product supply strategy, assuming that only a one distribution model considered, in which the manufacturer produces the product - the wholesale of the product to the retailer - the retailer sells the product to the individual customer. We first design the variables involved in the research and then based on this study, build a mathematical model of a retailer-personal customer product supply strategy under the false demand pattern.

Based on the demand function model of Petruzzi and Dada (1999) and the study of consumer product effectiveness by Chen and Wang (2012), we define the expression of the false demand function ${ }^{1}$.

$$
D_{F}=\frac{3}{2}\left(\frac{p-v p_{m}}{p_{m}(u-v)}-d_{0}\right)(a-b p+\varepsilon), \quad a, b>0, \varepsilon \in U\left(\mu, \sigma^{2}\right)
$$

In this case, we set $\varepsilon$ to obey a uniformly distributed function in the interval as [- $(a-b p), a-b p]$.

Then,

$$
f(\varepsilon)=\frac{1}{2(a-b p)}, \quad \varepsilon \in[-(a-b p), a-b p]
$$

Below we will analyze the optimal purchase volume and optimal pricing scheme of retailers in the false demand pattern from customer discount mode.

\subsection{Retailer's Profit Function}

In the operation of product supply Strategies, the manufacturer must first wholesale the product to the retailer. If the quantity $q$ of the wholesale product exceeds the customer's demand, there will be surplus products, and the remaining

\footnotetext{
${ }^{1}$ Zhuo, Z. Y., Yan, H, He, Y. (2019). Analysis of Retailer-to-Individual Customer Product Supply Strategies under a False Demand Pattern. To Paper.
} 
products also occupy the inventory space. Therefore, this phenomenon of oversupply is unable to achieve profit maximization. On the contrary, if the quantity purchased is less than the customer's demand, this shortage of supply has resulted in a profit leakage for the remaining customers. It is also impossible to maximize profits.

In the supply has outstripped demand phase, set the purchased quantity is $q$, the demand quantity is $p D(p, \varepsilon)$, and the remaining quantity $q-D(p, \varepsilon)$ is processed according to the cost per unit $h$. In this, $h \geq-c$ can take a negative value, which represents the residual of the unit product. So, in this paragraph, the retailer's profit is,

$$
\Pi(p, p)=p D(p, \varepsilon)-c q-h[q-D(p, \varepsilon)]
$$

In the same way, in the phase of supply less than demand, If the purchased quantity is $q$, the selling price is $p q$, and the loss part per unit is $S$. At this time, the retailer's profit is,

$$
\Pi(q, p)=p q-c q-S[D(p, \varepsilon)-q]
$$

So, we define the retailer's profit as:

\section{Parameter Indicator Design}

$$
\Pi(p, p)= \begin{cases}p D(p, \varepsilon)-c q-h[q-D(p, \varepsilon)], & q \geq D(p, \varepsilon) \\ p q-c q-S[D(p, \varepsilon)-q], & q<D(p, \varepsilon)\end{cases}
$$

According to the above-related variable function, we will construct a mathematical model of the retailer-individual customer product supply strategy under the false demand pattern. Before this, we first design the parameter indicators needed for modeling (table1):

Table 1. The list of the notation

SYMBOL

\begin{tabular}{c|c}
\hline$\alpha$ & The discounted price per product in the off-invoice model \\
$p$ & The retail price set by the retailer for the product \\
$q$ & The quantity ordered by the retailer to the manufacturer \\
$u$ & The practical performance of the product \\
$v$ & The utility function of the product \\
$w_{0}$ & The marginal cost of each unit produced by the manufacturer \\
$d_{0}$ & The weight' minimum value on practical performance of the product \\
$p_{m}$ & The product maximum performance or reserve price \\
$D_{F}$ & The false demand function \\
$\Pi_{R}$ & The retailer's profit \\
$I$ & Individual customer \\
\hline
\end{tabular}

\section{Mathematical Model Construction: Customer Discount Mode}

To increase sales and promote profit, retailers often have various preferential measures such as discounts, rebates, and tax rebates, etc. Suppose the retailer's purchase amount is $q$, the retail price of the product sold by the retailer to the customer is $p$, and the amount of the returnable tax give as $x$, and tax refund per as $T$. If the quantity of the merchandise exceeds this quota, the fee will refund to the customer.

When $q \leq x$, the profit of the retailer available is:

$$
\Pi_{R}=-w_{0} q+p \int_{-\infty}^{q} D_{F} f(\varepsilon) d \varepsilon+p \int_{q}^{+\infty} q f(\varepsilon) d \varepsilon
$$

When $q>x$, the profit of the retailer available is:

$$
\begin{aligned}
\Pi_{R}= & -w_{0} q+p \int_{-\infty}^{q} D_{F} f(\varepsilon) d \varepsilon+p \int_{q}^{+\infty} q f(\varepsilon) d \varepsilon \\
& -T\left(\int_{x}^{q} D_{F} f(\varepsilon) d \varepsilon+\int_{q}^{+\infty} q f(\varepsilon) d \varepsilon\right)
\end{aligned}
$$

In this supply mode, the retailer's optimal purchase volume and optimal pricing are only required to use the optimization method to derive the correlation function from the above formula and set its result is 0 . 
(1) When $q \leq x$, there is no return tax to customers, then

$$
\begin{aligned}
\Pi_{R} & =-w_{0} q+p \int_{0}^{q} \frac{3}{2}\left(\frac{p-v p_{m}}{p_{m}(u-v)}-d_{0}\right) \varepsilon \cdot \frac{1}{2(a-b p)} d \varepsilon+p \int_{q}^{2(a-b p)} q \cdot \frac{1}{2(a-b p)} d \varepsilon \\
& =\left(-w_{0}+p\right) q-\frac{p q^{2}}{2(a-b p)}+\frac{3 p q^{2}}{8(a-b p)}\left(\frac{p-v p_{m}}{p_{m}(u-v)}-d_{0}\right)
\end{aligned}
$$

Then,

$$
\frac{\partial \Pi_{R}}{\partial q}=\left(-w_{0}+p\right)-\frac{p q}{(a-b p)}+\frac{3 p q}{4(a-b p)}\left(\frac{p-v p_{m}}{p_{m}(u-v)}-d_{0}\right)=0
$$

Then,

$$
p q\left(\frac{3 p-3 v p_{m}}{p_{m}(u-v)}-3 d_{0}-4\right)=4\left(w_{0}-p\right)(a-b p)
$$

Solved,

$$
q=\frac{4\left(w_{0}-p+T\right)(a-b p)}{(p-T)\left(\frac{3 p-3 v p_{m}}{p_{m}(u-v)}-3 d_{0}-4\right)}
$$

$q$ is the retailer's optimal purchase amount. At this point, the retailer's optimal pricing formula is,

$$
\frac{\partial \Pi_{R}}{\partial p}=q-\frac{a q^{2}}{2(a-b p)^{2}}+\frac{3 q^{2}}{8(a-b p)^{2}} \cdot\left(\frac{2 a p-b p^{2}-a p_{m} v}{p_{m}(u-v)}-a d_{0}\right)=0
$$

Then,

$$
b\left(8 b-\frac{3 q^{2}}{p_{m}(u-v)}\right) p^{2}-2 a\left(8 b-\frac{3 q}{p_{m}(u-v)}\right) p+\left[8 a^{2}-a q^{2}\left(\frac{3 v p_{m}}{p_{m}(u-v)}+3 d_{0}+4\right)\right]=0
$$

Set,

$$
\begin{aligned}
& A=b\left(8 b-\frac{3 q^{2}}{p_{m}(u-v)}\right) ; \\
& B=-2 a\left(8 b-\frac{3 q}{p_{m}(u-v)}\right) ; \\
& C=8 a^{2}-a q^{2}\left(\frac{3 p_{m} v}{p_{m}(u-v)}+3 d_{0}+4\right)
\end{aligned}
$$

Solved,

$$
p=\frac{-B+\sqrt{B^{2}-4 A C}}{2 A}
$$

$p$ is the retailer's optimal pricing.

(2) When $q>x$, there is a tax refund for products purchased by customers, then 


$$
\begin{aligned}
\Pi_{R}= & -w_{0} q+p \int_{0}^{q} \frac{3}{2}\left(\frac{p-v p_{m}}{p_{m}(u-v)}-d_{0}\right) \varepsilon \cdot \frac{1}{2(a-b p)} d \varepsilon+p \int_{q}^{2(a-b p)} q \cdot \frac{1}{2(a-b p)} d \varepsilon \\
& -T \int_{x}^{q} \frac{3}{2}\left(\frac{p-v p_{m}}{p_{m}(u-v)}-d_{0}\right) \cdot \varepsilon \cdot \frac{1}{2(a-b p)} d \varepsilon-T \int_{q}^{2(a-b p)} q \frac{1}{2(a-b p)} d \varepsilon \\
= & \left(-w_{0}+p-T\right) q-\frac{(p-T) q^{2}}{2(a-b p)}+\frac{3(p-T) q^{2}+3 T x^{2}}{8(a-b p)}\left(\frac{p-v p_{m}}{p_{m}(u-v)}-d_{0}\right)
\end{aligned}
$$

Then,

Then,

$$
\frac{\partial \Pi_{R}}{\partial q}=\left(-w_{0}+p-T\right)-\frac{(p-T) q}{(a-b p)}+\frac{3(p-T) q}{4(a-b p)}\left(\frac{p-v p_{m}}{p_{m}(u-v)}-d_{0}\right)=0
$$

Solved,

$$
(p-T) q\left(\frac{3 p-3 v p_{m}}{p_{m}(u-v)}-3 d_{0}-4\right)=4\left(w_{0}-p+T\right)(a-b p)
$$

$$
q=\frac{4\left(w_{0}-p+T\right)(a-b p)}{(p-T)\left(\frac{3 p-3 v p_{m}}{p_{m}(u-v)}-3 d_{0}-4\right)}
$$

$q$ is the retailer's optimal purchase amount. At this point, the retailer's optimal pricing formula is,

$$
\begin{aligned}
\frac{\partial \Pi_{R}}{\partial p} & =q-\frac{q^{2}(a-b T)}{2(a-b p)^{2}}+\frac{3}{8(a-b p)^{2}} \cdot\left(\frac{2 p q^{2}-v p_{m} q^{2}-T q^{2}+T x^{2}}{p_{m}(u-v)}-d_{0} q^{2}\right) \\
& +\frac{3 b(p-T) q^{2}+3 b T x^{2}}{8(a-b p)^{2}}\left[\frac{p-v p_{m}}{p_{m}(u-v)}-d_{0}\right]=0
\end{aligned}
$$

Then,

$$
\begin{aligned}
& b q\left(8 b-\frac{3 q}{p_{m}(u-v)}\right) p^{2}-16 a b q-\frac{6 q^{2}-3 b v p_{m} q^{2}+3 b T q^{2}-3 b T x^{2}}{p_{m}(u-v)}-3 b q^{2} d_{0}+8 a^{2} q \\
& -4 q^{2}(a-b T)-3\left(\frac{T\left(q^{2}-x^{2}\right)+v p_{m} q^{2}}{p_{m}(u-v)}+d_{0} q^{2}\right)+3 b T\left(q^{2}-x^{2}\right)\left(\frac{v p_{m}}{p_{m}(u-v)}+d_{0}\right)=0
\end{aligned}
$$

Set,

$$
\begin{aligned}
& A=b q\left(8 b-\frac{3 q}{p_{m}(u-v)}\right) \\
& B=16 a b q-\frac{6 q^{2}-3 b v p_{m} q^{2}+3 b T q^{2}-3 b T x^{2}}{p_{m}(u-v)}-3 b q^{2} d_{0} \\
& C=8 a^{2} q-4 q^{2}(a-b T)-3\left(\frac{T\left(q^{2}-x^{2}\right)+p_{m} v q^{2}}{p_{m}(u-v)}+d_{0} q^{2}\right)+3 b T\left(q^{2}-x^{2}\right)\left(\frac{v p_{m}}{p_{m}(u-v)}+d_{0}\right)
\end{aligned}
$$

Solved, 


$$
p=\frac{-B+\sqrt{B^{2}-4 A C}}{2 A}
$$

$p$ is the retailer's optimal pricing.

\section{Conclusions}

This article considers the retailer's optimal product supply strategy for the customer discount mode under the false demand pattern. It solves the retailer's optimal product pricing and optimal production volume under this supply model. The research results not only provide an essential basis for the retailer's product supply strategy when the supply is higher than the demand, and the supply is less than the demand. And also assist the retailer in making more accurate product sales decisions for individual customers under the false demand pattern. In the future, we can consider using the multiplication form of the demand function to perform functional modeling of different demand patterns. By comparing the two types of profit function models, and analyzed the difference between the demand function in the addition form and the demand function in the multiplication form, to further improve the theoretical research of different demand patterns in the consumer goods supply chain.

\section{Acknowledgements}

The author thanks the reviewers very much for their careful review and valuable comments.

\section{References}

Abdel-Aal, M. A., \& Selim, S. Z. (2019). Robust optimization for selective newsvendor problem with uncertain demand. Computers \& Industrial Engineering, 135, 838-854. https://doi.org/10.1016/j.cie.2019.06.047

Chen, Q., \& Wang, Q. (2012). A bertrand equilibrium model based on evolvement and innovation of product function. Systems Engineering, 30(2), 9-14.

Cohen, M. C., Lobel, R., \& Perakis, G. (2016). The impact of demand uncertainty on consumer subsidies for green technology adoption. Management Science, 62(5), 1235-1258. https://doi.org/10.1287/mnsc.2015.2173

Crawford, M. (2004). The world in a shopping mall. In Sorkin. M. (Ed), Variations on a theme park: The new American city and the end of public space (pp.3-30). New York: Hill \& Wang.

Bustinza, F. O., Parry, C. G., \& Vendrell-Herrero, F. (2013). Supply and demand chain management: The effect of adding services to product offerings. Supply Chain Management: An International Journal, 18(6), 618-629.https://doi.org/10.1108/SCM-05-2013-0149

Gause, D. C., \& Weinberg, G. M. (2011). Exploring requirements: quality before design. New York: Dorset House Pub.

Kim, Y., \& Krishnan, R. (2015). On product-level uncertainty and online purchase behavior: An empirical analysis. Management Science, 61(10), 2449-2467. https://doi.org/10.1287/mnsc.2014.2063

Kisialiou, Y., Gribkovskaia, I., \& Laporte, G. (2019). Supply vessel routing and scheduling under uncertain demand. Transportation Research Part C: Emerging Technologies, 104, 305-316. https://doi.org/10.1016/j.trc.2019.04.011

Maslow, A. H. (1943). A theory of human motivation. Psychological Review, 50(4), $370-96$. https://doi.org/10.1037/h0054346

Marcuse, H. (1991). One-dimensional man: Studies in the ideology of advanced industrial society (2nd ed.). Gibraltar: Beacon Press. https://doi.org/10.1037/11144-000

Marx, K., \& Engels, F. (1965). The german ideology. London, England.

McClelland, D. C., Atkinson, J. W., Clark, R. A., \& Lowell, E. L. (1953). Century psychology series. The achievement motive. East Norwalk, CT, US: Appleton-Century-Crofts.

Moradi, A., Razmi, J., Babazadeh, R., \& Sabbaghnia, A. (2018). An integrated Principal Component Analysis and multi-objective mathematical programming approach to agile supply chain network design under uncertainty. Journal of Industrial \& Management Optimization, 433-459.

Morgenstern, O. (1948). Demand theory reconsidered. The Quarterly Journal of Economics, 62(2), $165-201$. https://doi.org/10.2307/1883219

Nazari, M., \& Foroud, A. A. (2013). Optimal strategy planning for a retailer considering medium and short-term decisions. International journal of Electrical power \& Energy systems, 45(1), 107-116. https://doi.org/10.1016/j.ijepes.2012.08.068

Özer, Ö., \& Uncu, O. (2015). Integrating dynamic time-to-market, pricing, production and sales channel decisions. European Journal of Operational Research, 242(2), 487-500. https://doi.org/10.1016/j.ejor.2014.09.001 
Petruzzi, N. C., \& Dada, M. (1999). Pricing and the newsvendor problem: A review with extensions. Operations research, 47(2), 183-194. https://doi.org/10.1287/opre.47.2.183

Rachlin, H., Green, L., Kagel, J. H., \& Battalio, R. C. (1976). Economic demand theory and psychological studies of choice. In Psychology of Learning and Motivation (Vol. 10, pp. 129-154). Academic Press. https://doi.org/10.1016/S0079-7421(08)60466-1

Weskamp, C., Koberstein, A., Schwartz, F., Suhl, L., \& Voß, S. (2019). A two-stage stochastic programming approach for identifying optimal postponement strategies in supply chains with uncertain demand. Omega, 83, 123-138. https://doi.org/10.1016/j.omega.2018.02.008

Xie, Y., He, W., Ching, W. K., Tai, A. H., Ip, W. H., Yung, K. L., \& Song, N. (2019). Optimal advertising outsourcing strategy with different effort levels and uncertain demand. International Journal of Production Research, 1-20. https://doi.org/10.1080/00207543.2019.1641235

Yuan, H., Gómez, M. I., \& Rao, V. R. (2013). Trade promotion decisions under demand uncertainty: A market experiment approach. Management Science, 59(7), 1709-1724. https://doi.org/10.1287/mnsc.1120.1652

Zhang, B., Duan, D., \& Ma, Y. (2018). Multi-product expedited ordering with demand forecast updates. International Journal of Production Economics, (206), 196-208. https://doi.org/10.1016/j.ijpe.2018.09.034

\section{Copyrights}

Copyright for this article is retained by the author(s), with first publication rights granted to the journal.

This is an open-access article distributed under the terms and conditions of the Creative Commons Attribution license (http://creativecommons.org/licenses/by/4.0/). 